\title{
Effects induced by high and low intensity laser plasma on SiC Schottky detectors
}

\author{
${\text { Antonella } \text { Sciuto }^{1 *} \text {, Lorenzo Torrisi }}^{2}$, Antonino Cannavò ${ }^{2}$, Massimo Mazzillo ${ }^{3}$ and Lucia Calcagno ${ }^{4}$ \\ ${ }^{1}$ CNR-IMM, VIII strada n ${ }^{\circ}$, 95121 Catania, Italy \\ ${ }^{2}$ Dip.to Scienze Fisiche-MIFT, Università di Messina, Agata (ME),Italy \\ ${ }^{3}$ STMicroelectronics, Str.le Primosole 50, Catania, Italy \\ ${ }^{4}$ Dip.to di Fisica ed Astronomia, Università di Catania, Italy
}

\begin{abstract}
Silicon-Carbide detectors are extensively employed as diagnostic devices in laser-generated plasma, allowing the simultaneous detection of photons, electrons and ions, when used in time-of-flight configuration. The plasma generated by high intensity laser $\left(10^{16} \mathrm{~W} / \mathrm{cm}^{2}\right)$ producing high energy ions was characterized by $\mathrm{SiC}$ detector with a continuous front-electrode, and a very thick active depth, while SiC detector with an Interdigit front-electrode was used to measure the low energy ions of plasma generated by low intensity laser $\left(10^{10} \mathrm{~W} / \mathrm{cm}^{2}\right)$. Information about ion energy, number of charge states, plasma temperature can be accurately obtained. However, laser exposure induces the formation of surface and bulk defects whose concentration increases with increasing the time to plasma exposure. The surface defects consist of clusters with a main size of the order of some microns and they modify the diode barrier height and the efficiency of the detector as checked by alpha spectrometry. The bulk defects, due to the energy loss of detected ions, strongly affect the electrical properties of the device, inducing a relevant increase of the leakage (reverse) current and decrease the forward current related to a deactivation of the dopant in the active detector region.
\end{abstract}

\section{Introduction}

Silicon Carbide $(\mathrm{SiC})$ detectors are now-days widely employed for the monitoring of ionizing radiations. Due to the physical properties of the material, these detectors can be used at high temperatures and in high level radioactive ambient [1-3]. Irradiation of $\mathrm{SiC}$ detectors with neutrons, protons and heavy ions evidenced the higher radiation hardness of these devices with respect to $\mathrm{Si}$ detectors, principally thanks to higher atomic displacement energy of the $\mathrm{SiC}$. It has been shown $[4,5]$ that $\mathrm{SiC}$ detectors retain their detection properties (such as efficiency and resolution) up to irradiation doses as high as 10 MGy [6,7]. These remarkable results allowed to widely use $\mathrm{SiC}$ detectors in nuclear and plasma physics $[8,9]$.

In the last decade, they are extensively used as diagnostic devices in laser generated plasma for both high and low intensity laser. In this field they found large application thanks to the blindness to visible light emitted from pulsed laser and to the visible component of the plasma itself. In particular, they are used in Time of Flight (TOF) configuration as this technique allows the simultaneous detection of photons, electrons and ions emitted from laser plasma; the energy of radiation can be measured with high accuracy and the detector show a high efficiency and sensitivity together with a fast response [10-12].

In our recent works, we have shown [13] that $\mathrm{SiC}$ detectors with two different front-electrode geometries allow to detect ions in a wide range of energy from few $\mathrm{keV}$ to $10 \mathrm{MeV}$. The detectors with a continuous frontelectrode and a thick active region are able to measure the high energy ions generated by high intensity $\left(10^{16}\right.$ $\left.\mathrm{W} / \mathrm{cm}^{2}\right) n s$ or $f s$ pulsed laser. Instead $\mathrm{SiC}$ devices with an interdigit front-electrode can be employed to detect the low energy of the ions emitted by low intensity $\left(10^{10}\right.$ $\mathrm{W} / \mathrm{cm}^{2}$ ) laser.

Some of us demonstrated, in a recent paper [14], that by using both these detectors we can realize a telescope ion detector to measure the energy and the energy loss of ions generated by high intensity lasers. The proposed telescope system was based on the use of a M-SiC device with thick active detection layer to measure the total ion energy and of an I-SiC device with thinner active detection layer to measure the ion's energy loss. By plotting the ion energy versus the ion energy loss, it is possible to distinguish the different detected ion species.

Furthermore, due to their micrometric size, these detectors are very promising for the realization of

* Corresponding author: antonella.sciuto@imm.cnr.it 
pixilated sensors for the measurements of radiation spatial distribution $[15,16]$.

Although SiC detectors are very useful for the laser plasma characterization, their use could be limited by the damage produced during long-time exposure to plasma. In particular, we must distinguish between a bulk damage consisting of defects created in the detector active depth and due to the ion energy loss and a surface damage due to the debris and defect clusters generated by plasma at the device surface [17].

In the present paper we report about damage produced in both type of detectors (M-SiC and I-SiC) after plasma exposure. The surface damage is monitored by optical microscopy and the increases of defect concentration with laser exposure time was monitored. Moreover, the effect of damage generated by low and high intensity laser on the electrical characteristics of the devices and on the detector performances was examined.

\section{Experimental}

SiC detectors based on Schottky diode technology with different active volume and with two different front electrode structures were examined in this work. They were fabricated at CNR-IMM-Catania (M-SiC) and STMicroelectronics-Catania (I-SiC) facilities and are widely described, from the electrical and the detection point of view, in previous work of authors of the present manuscript [13]. The first proposed detector (M-SiC), is a continuous metal vertical Schottky device, fabricated on n-type $4 \mathrm{H}-\mathrm{SiC}$ epitaxial layer, $80 \mu \mathrm{m}$ thick with a $2 \times 10^{14} \mathrm{~cm}^{-3} n$-dopant concentration, grown on $n$-type heavily doped $\left(\sim 6 \times 10^{18} \mathrm{~cm}^{-3}\right)$ substrate.

The second type (I-SiC), is an interdigit metal vertical Schottky device, fabricated on $n$-type $4 \mathrm{H}-\mathrm{SiC}$ epitaxial layer, $4 \mu \mathrm{m}$ thick with a $1 \times 10^{14} \mathrm{~cm}^{-3} n$-dopant concentration grown on $n$-type heavily doped substrate. In both devices, the ohmic contacts on the sample back side were formed by sputtering of a $100 \mathrm{~nm}$ thick nickel (Ni) film, followed by a rapid annealing at $1000{ }^{\circ} \mathrm{C}$ in $\mathrm{N}_{2}$; similarly, the front electrode was obtained by $\mathrm{Ni}$ (100 nm thick) deposition, photolithographic patterning, selective metal etch and rapid thermal process at $700{ }^{\circ} \mathrm{C}$ in $\mathrm{N}_{2}$ environment for the formation of the $\mathrm{Ni}_{2} \mathrm{Si}$ layer (200 $\mathrm{nm}$ thick) and of the optimal Schottky electrode [18].

The M-SiC diodes have a square geometry with a total area of about $4 \mathrm{~mm}^{2}$ and was polarised at $600 \mathrm{~V}$ in order to deplete all the $80 \mu \mathrm{m}$ active region. The I-SiC have a total area of about $8 \mathrm{~mm}^{2}$ and an area of about $6 \mathrm{~mm}^{2}$ directly exposed to the impinging radiation $(75 \%$ of fill factor being the single $\mathrm{Ni}_{2} \mathrm{Si}$ strip width of about $3 \mu \mathrm{m}$ and the distance between contiguous front metal stripes of about $10 \mu \mathrm{m}$ ). It was polarised at $20 \mathrm{~V}$, bias enough to deplete the $4 \mu \mathrm{m}$ active layer. More details of both detectors are given in $[19,20]$.

In this work we report measurements at low laser intensity, performed at INFN-Laboratori Nazionali del Sud in Catania and at high laser intensity, performed at the PALS facility in Prague. In the first case we employed a Nd:YAG laser operating at $1064 \mathrm{~nm}$ wavelength, with a $9 \mathrm{~ns}$ pulse duration, $200 \mathrm{~mJ}$ laser energy and $2 \times 10^{10} \mathrm{~W} \mathrm{~cm}^{-2}$ pulse intensity. In the second case an Iodine laser operating in single pulse regime at $1315 \mathrm{~nm}$ wavelength, with $300 \mathrm{ps}$ pulse duration, $600 \mathrm{~J}$ laser energy and $2 \times 10^{16} \mathrm{~W} \mathrm{~cm}^{-2}$ pulse intensity was used.

The detectors efficiency and resolution were checked by high energetic alpha particles using a radioactive source composed by three peaks, ${ }^{239} \mathrm{Pm}(5.15 \mathrm{MeV}),{ }^{241} \mathrm{Am}$ $(5.48 \mathrm{MeV})$ and ${ }^{241} \mathrm{Cm}(5.80 \mathrm{MeV})$.

\section{Results}

The M-SiC and I-SiC detectors were used to monitor the radiation emitted by plasma generated by high and low intensity laser. Typically, high intensity laser generates high energy ions, and their detection needs very thick active region detector, as our $\mathrm{M}-\mathrm{SiC}$ diode. In Fig. 1 is shown a typical TOF spectrum acquired at PALS facility at $10^{16} \mathrm{~W} / \mathrm{cm}^{2}$ laser intensity by using an $\mathrm{M}-\mathrm{SiC}$ device. The spectrum was obtained by irradiating a thin foil of Polyethylene $(20 \mu \mathrm{m}$ thick $)$, and detecting radiation at $0^{\circ}$ in forward direction with respect to laser incidence. The peak in the $0-10 \mathrm{~ns}$ region is related to hard X-ray radiation (from few $\mathrm{keV}$ up to few tens of $\mathrm{keV}$ ) emitted during and after the laser-target interaction.

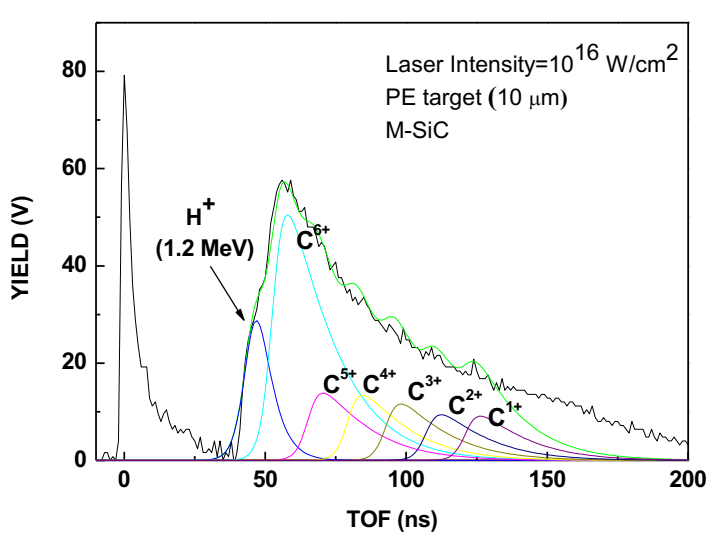

Fig.1. Time of flight spectrum of the plasma generated by high intensity pulsed laser irradiating a PE, $20 \mu \mathrm{m}$ thick target, detected by M-SiC.

The spectrum structure between 10 and $30 \mathrm{~ns}$ is ascribable to XUV photons due to electron bremsstrahlung or to plasma electrons. The most interesting region of the spectrum is the one in the TOF range between 30 and $200 \mathrm{~ns}$, due to the energetic ions of the plasma, from protons to carbon of different charge states and slower $\mathrm{C}_{\mathrm{x}} \mathrm{H}_{\mathrm{y}}$ groups, detected at time of flight higher than $150 \mathrm{~ns}$. The $\mathrm{SiC}$ detector allows separation of the photopeak from the ion component, observable as overlap of many different peaks. The fit of this spectrum with CBS (Coulomb-Boltzman-Shifted) model [21] allows the de-convolution of the ion peak with single components: the faster peak is related to the protons and it is followed by peaks related to $\mathrm{C}$ ions with different 
charge states. The fit allows to evaluate some important plasma parameters, such as the equivalent temperature and the acceleration energy. We obtained an equivalent temperature of $54 \mathrm{keV}$, and an energy of $1.2 \mathrm{MeV}$ for charge state, in good agreement with data reported in literature [22].

At low intensity laser, ions coming from laser-target interaction do not have enough energy to overcome the metallization layer of $\mathrm{M}-\mathrm{SiC}$ detector, so the generated plasma can be monitored only by detector with active layer directly exposed to irradiation, that is the I-SiC. A typical example of TOF spectrum measured with an I$\mathrm{SiC}$ detector by irradiating an $\mathrm{Al}$ target is shown in Fig.2. The spectrum of the Al target evidences a broad peak at $5.5 \mu$ s resulting in an energy of $1.2 \mathrm{keV}$, value typical of ions generated by low intensity lasers [23]. In this case, due to the low energy $(200 \mathrm{~mJ})$ of laser only the single charge state of $\mathrm{Al}$ is generated [24].

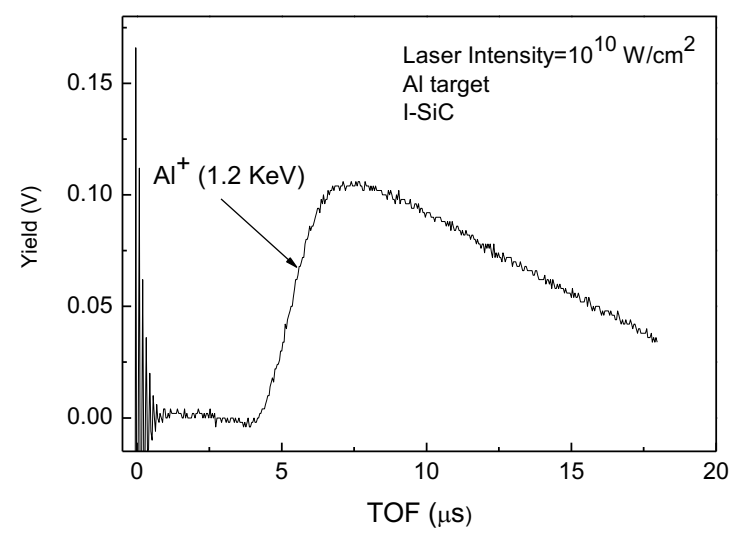

Fig. 2. Time of flight spectrum of the plasma generated by low intensity pulsed laser irradiating a Al target detected by I$\mathrm{SiC}$.

However, plasma exposure of $\mathrm{SiC}$ detectors generated some surface damage as evidenced in Fig.3, where are shown the photographs of I-SiC (Fig.3a) and M-SiC (Fig.3c) detector surface after laser irradiation. The I-SiC were exposed to low intensity laser in Catania to a number of about 100 shots, while the M-SiC were exposed to high intensity laser at PALS laboratory in Prague to a number of about 300 shots. Before irradiation, both detectors show a clean surface with a delineated geometry, while after plasma exposure the surface results covered by debris consisting of micrometric clusters. The cluster size distribution is quite large in both case, however for the I-SiC the main cluster size (Fig. $3 \mathrm{~b}$ ) is $8 \mu \mathrm{m}$, while for the M-SiC (Fig.3d) is $18 \mu \mathrm{m}$. The increase of exposure time to the plasma clearly enhances the total number of such clusters, but their morphology and size depend on plasma parameters such as temperature, density, angular distribution of emitted particles and also on the detector position with respect to target as distance and angle. In particular, the wavelength and the intensity of laser influence the phothermal and photochemical ablation regime and at high intensity larger molecular or clusters are formed during plasma generation [25].

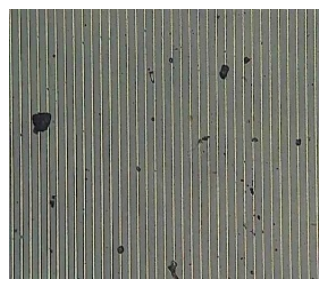

(a)

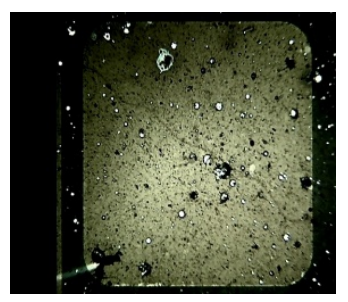

(c)

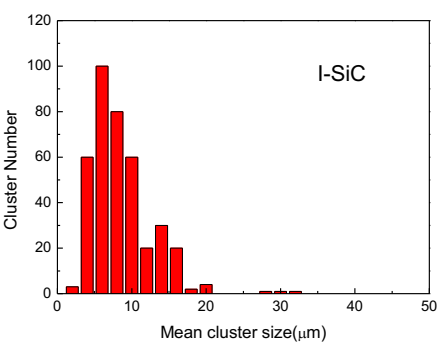

(b)

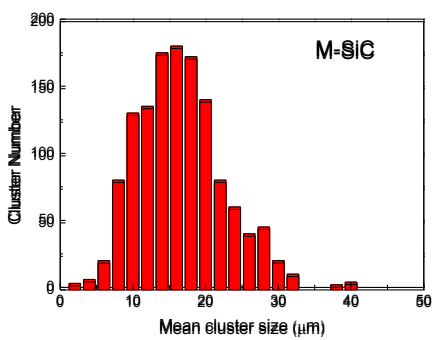

(d)
Fig. 3. Optical microscopy image (a) and cluster size distribution (b) of a portion of the I-SiC detector after exposure to low intensity laser. Optical microscopy image (c) and cluster size distribution (d) of $\mathrm{M}-\mathrm{SiC}$ detector after exposure to high intensity laser.

The presence of damage has a considerable influence on the device electrical characteristics and in Fig.4 are shown the I-V measurements of I-SiC before and after laser exposure. The reverse current (Fig.4a) of detector before plasma exposure is very low, about $5 \times 10^{-11} \mathrm{~A}$, and increases of more than two order of magnitude after laser irradiation.

The forward characteristic (Fig.4b) of as prepared device evidences its excellent rectifying properties; it exhibits a region of linearity at low bias value $(<1.5 \mathrm{~V})$ and a saturation at higher voltage, where the device behavior is dominated by the series resistances. The fit of this curve gives a value of $1.65 \mathrm{eV}$ for the Schottky barrier height and of 1.05 for the ideality factor, close to literature values [26].

After laser exposure the linear region of the curves gets small and an increase of current at low bias occurs. In addition, a small decrease of current in the saturation region is observed related to the increase of series resistance of device [27].

Similar results are obtained in $\mathrm{M}-\mathrm{SiC}$ detector irradiated at PALS whose I-V characteristics are reported in Fig.5. The reverse current after laser exposure (Fig.5a) increases of about four order of magnitude, while the forward characteristics shows a quite strange behavior: at low bias $(<0.3 \mathrm{~V})$ it increases and then saturates at higher voltage.

The comparison between the forward I-V curves of I-SiC and $\mathrm{M}-\mathrm{SiC}$ after laser exposure, shows that the $\mathrm{M}-\mathrm{SiC}$ devices suffered a larger increase of current at low bias and a further current decrease at high bias. This difference is due both to the larger amount of damage and to the different type of damage produced in the M$\mathrm{SiC}$. 

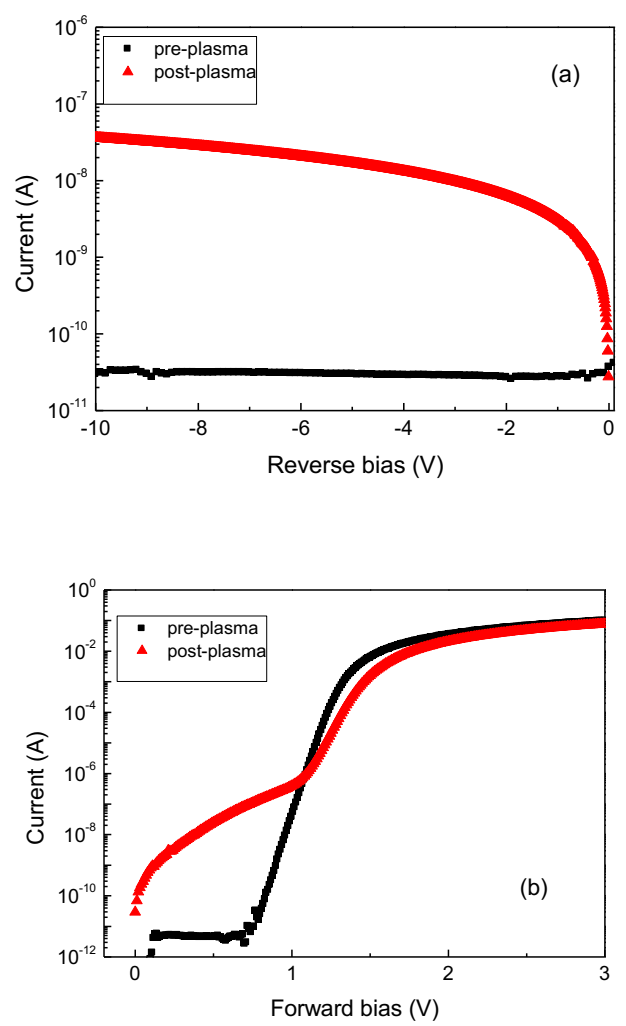

Fig. 4. Reverse (a) and forward (b) I-V characteristics of I-SiC detector before and after laser exposure.

In fact, we must underline that plasma exposure together to the formation of already described surface damage, induces the formation of defect localized into the active region of the device and due to penetrating ions. The energy loss of the ions produces the formation of point defects (interstitial, vacancies, antisite) and complex defects (dislocation, stacking faults, etc) localized into a depth close to the ion range. Because the laser produces ions with different charge states (i.e. energies), the defects are distributed into the entire irradiated depth. As already reported in literature [27] ion irradiation of $\mathrm{SiC}$ Schottky diodes is responsible of the increase of leakage current and of the decrease of forward current at high bias. In particular, point and complex defects induce the formation of deep levels in the forbidden gap which act as generation centers increasing the leakage current. At the same time, these defects imply a deactivation of dopant in the irradiated region, with a consequente increase of series resistance and decrease of forward current at high bias. This effect in the M-SiC is larger with respect to I-SiC, because of the different involved ion range, which is of the order of $40-50 \mu \mathrm{m}$ in the M$\mathrm{SiC}$ and of some hundred of Angstrom in I-SiC.

The increase of forward current at low bias can be, instead, ascribed to the surface damage as the presence of clusters and debris modifies the Schottky barrier, producing an inhomogenous barrier which is composed by a distribution of nanometric size "patches" with a low barrier height $(<1 \mathrm{eV})$ enclosed in a uniform high barrier background (1.65 eV barrier height in un-exposed diode). The formation of paths of low barrier height gives a strong contribution to the extra current measured at low bias [28].
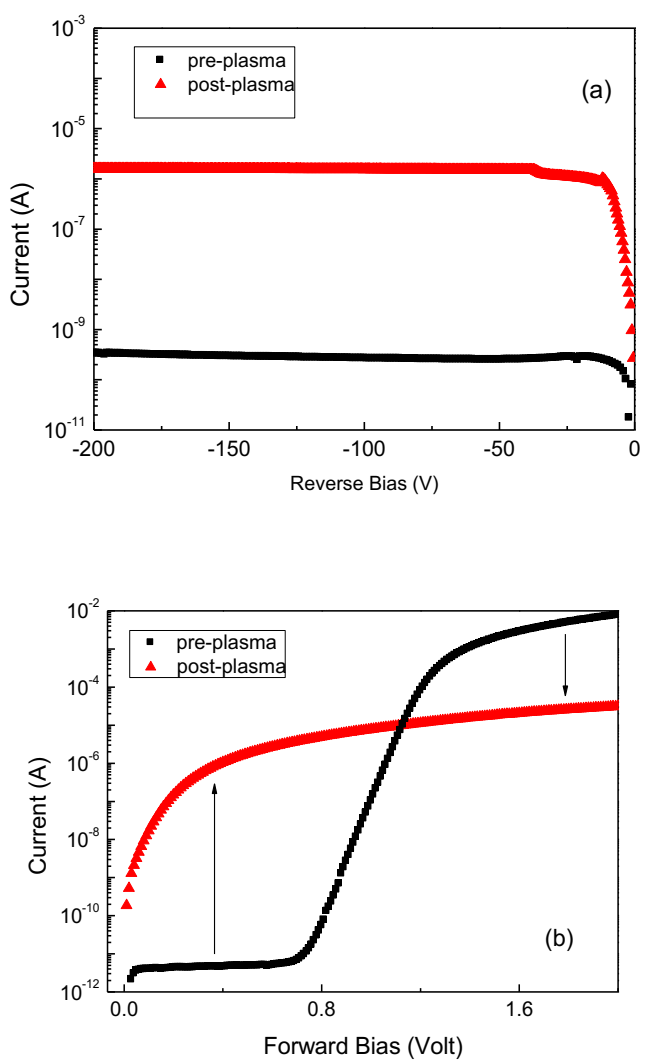

Fig. 5. Reverse (a) and forward (b) IV characteristics of M-SiC detector before and after laser exposure.

The effect of damage on the detection properties of the $\mathrm{M}-\mathrm{SiC}$ detector was checked by a three peaks alpha source. The I-SiC cannot be used for these measurements because the range of alpha particles (18 $\mu \mathrm{m})$ is higher than the active region thickness $(4 \mu \mathrm{m})$.

The alpha spectra measured in as-prepared M-SiC diode and after two different number of laser exposures (300 and 400 shots), acquired during the same slot, are shown in Fig.6. The detected yield of all peaks decreases after exposition, and it reaches a value of about $30 \%$ after a number of laser shots of about 400 .

This effect is mainly related to the coverage of surface by debris: the micrometric clusters induce a decrease of useful detector surface and a consequently reduction of the detection signal. In addition, a worsening of resolution from $0.9 \%$ to $1.5 \%$ is measured for the main peak $(5.15 \mathrm{MeV})$, which is related both to bulk defects and to energy straggling due to surface micrometric clusters. In order to evaluate the fluence impinging on the detector surface during laser exposure, we can take into account that an average fluence of $10^{14} \mathrm{ion} / \mathrm{cm}^{2}$ is produced by a single shot [17], then after 400 shots a total fluence of $4 \times 10^{16} \mathrm{ion} / \mathrm{cm}^{2}$ arrives on the detector surface. It is difficult to evaluate the absorbed dose 
(MGy) due to the different ion energies arriving simultaneously on the detector, however this fluence value is rather high and evidences the radiation hardness of these devices.

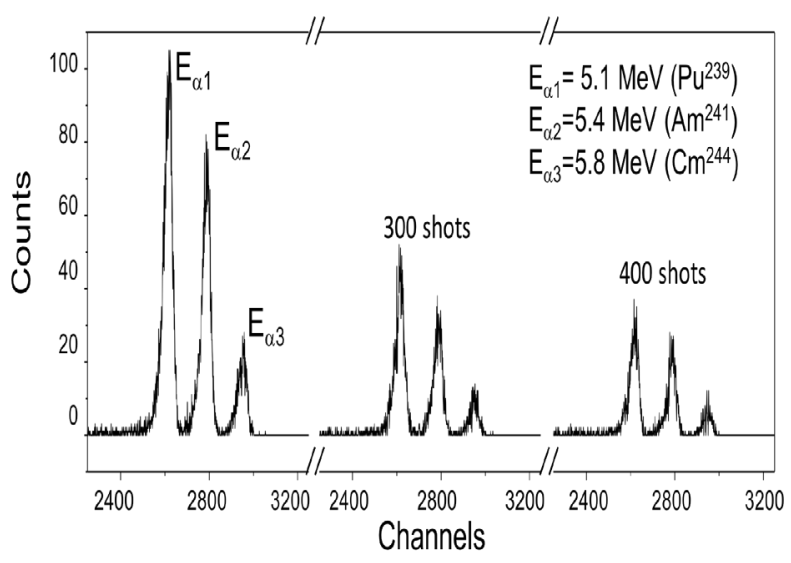

Fig.5: Three peaks alpha source spectra detected with $\mathrm{M}-\mathrm{SiC}$ before and after two different numbers of laser shots.

\section{Conclusions}

In the present paper we report some aspects regarding the damage induced in $\mathrm{SiC}$ detectors when exposed for long time to laser generated plasma. Two different detectors were tested. The first detector is an interdigit front electrode device where a fraction of surface is directly exposed to irradiation and then it is able to monitor the low energy ions generated by low intensity laser. To detect the high energy ions generated by high intensity laser a continuous front electrode diode with a thick epitaxial layer was used: this second detector is able to detect proton and ion of different charge states and to extract important plasma parameters, as temperature and ion acceleration. Laser exposure of both detectors produces some damage consisting in two types of defects. The first type is localized in the detector surface and consists in ablated and evaporated target material producing micrometric clusters: the presence of these clusters induces the formation of an inhomogeneous barrier and reduces the effective detector area with a decrease of detector efficiency. The second type of defects are points or complex defects generated by the energy loss of the different plasma ions and are localized in the active detector region: these defects induces an increase of leakage current and a deactivation of semiconductor dopant.

However, we like to underline that these effects are observed after long exposure time, highlighting the high radiation hardness of $\mathrm{SiC}$ detectors.

\section{Bibliography}

1. E.V. Kalinina, A.M.Ivanov, N.B.Strokan, Tech. Phys.Lett. 34, 210 (2008)

2. F.H. Ruddy, J.G.Seidel, Nucl.Instr.Meth. B 263, 163 (2007)

3. G. Bertuccio, R.Casiraghi, A.Cetronio, C.Lanzieri and F.Nava, Nucl.Instr.Meth.A 522,413 (2004)
4. F.Moscatelli, A.Scorzoni, A.Poggi, M.Bruzzi, S.Sciortino, S.Lagomarsino, G.Wagner, and R.Nipoti, IEEE Trans.on Nucl. Sci.53,1557 (2006)

5. S.Sciortino, F.Harties, S.Lagomarsino, F.Nava, M.Brianzi, V.Cindro, C.Lanzieri, and P.Vanni, Nucl.Instr.Meth. A 552, 138 (2005)

6. F. Nava, G. Bertuccio, A. Cavallini and E. Vittone, Meas. Sci. Technol. 19, 102011 (2008)

7. S.Sciortino, F.Harties, S.Lagomarsino, F.Nava, M.Brianzi, V.Cindro, C.Lanzieri, and P.Vanni, Nucl.Instr.Meth. A 552, 138 (2005)

8. G.Raciti, M.De Napoli, F.Giacoppo, E.Rapisarda and C.Sfienti, Nucl. Phys. A 834,784 (2010)

9. L.Torrisi, L.Calcagno, D.Giulietti, M.Cutroneo, M.Zimbone, J.Skala, Nucl.Instr.Meth., 355, 221 (2015)

10. L.Calcagno, P.Musumeci, M.Zimbone and F.La Via, Rad. Effects and Defects in Solids 4, 303 (2015)

11. M.Cutroneo, P.Musumeci, M.Zimbone, L.Torrisi, F.La Via, D.Margarone, A.Velyan, J.Ullschmied and L.Calcagno, J.Mat. Research 28, 87 (2013)

12. L.Torrisi, L.Calcagno, D. Giulietti, M.Cutroneo, M.Zimbone and J.Skala, Nucl.Instr.Meth. B 355, $221(2015)$

13. A.Sciuto, L.Torrisi, A.Cannavò, M.Mazzillo and L.Calcagno, J.Electronic Mater. (In Press)

14. L.Torrisi and A.Cannavò, IEEE Trans. Electron Dev. 63, 4445 (2016)

15. A.Sciuto, F.Roccaforte, S.Di Franco, V.Ranieri, S.F.Liotta, S.Billotta, G.Bonanno and M.Belluso, Mat.Sci.Forum 556, 945 (2007)

16. H.Shuoben, P.Hellström, C.M.Zetterling and M.Östling, IEEE Electr. Dev. Lett. 37, 1594 (2016)

17. L.Torrisi and A.Cannavò Journal of Instr. 11, 05009 (2016)

18. F.Roccaforte, F.La Via, V.Raineri, L.Calcagno, F.Mangano, J.App. Phys., 96, 4313 (2004)

19. A.Sciuto, L.Torrisi, A.Cannavò, G.Ceccio, P.Musumeci, M.Mazzillo, L.Calcagno, Vacuum, 131, 170 (2016)

20. P.Musumeci, M.Cutroneo, L.Torrisi, A.Velyan, M.Zimbone, L.Calcagno, Physica Scripta T161, 14021 (2014)

21. L. Torrisi, Radiat. Eff. Defect Solids 171, 34 (2016)

22. L. Torrisi, S. Gammino, L. Andò, L. Laska, J. Krasa, K. Rohlena, J. Ullschmied, J. Wolowski, J. Badziak, and P. Parys, J. Appl. Phys. 99, 083301 (2006)

23. L.Torrisi, S.Gammino, L.Andò and L.Laska, J.Appl.Phys. 91, 4685 (2002)

24. L. Torrisi, F. Caridi, A. Picciotto, A. Borrielli, Nucl.Instr.Meth.B 252 (2006) 183

25. L.Torrisi, A.Borrielli and D.Margarone, Nucl.Instr.Meth. B 255 (2007) 373

26. F.Roccaforte, F.La Via, V.Raineri, R.Pierobon, E.Zanoni, J.Appl.Phys. 93 (2003) 9137

27. F.Roccaforte, S.Libertino, V.Raineri, A.Ruggiero, V.Massimino and L.Calcagno J.Appl.Phys. 99, 13515 (2006)

28. L.Calcagno, A. Ruggiero, F.Roccaforte and F.La Via, J.Appl.Phys. 98,23713 (2005) 